\title{
Ciudad aumentada y pandemia El habitar en el Orden Digital
}

\section{P. Sebastián Cortez Oviedo}

Arquitecto, doctor en Arquitectura con formación en antropología social, especializado en mediatización urbana y espacios públicos. Becario posdoctoral del CONICET. Miembro investigador del INVIHAB-IDH CONICET, Facultad de Arquitectura, Urbanismo y Diseño, Universidad Nacional de Córdoba; Núcleo Inteligencia artificial, Sociedad y Comunicación, ICEI, Universidad de Chile; Equipo I-Polis, Instituto de Investigaciones Gino Germani, Facultad de Ciencias Sociales, Universidad de Buenos Aires. (INVIHAB - IDH CONICET).

pcortezoviedo@unc.edu.ar

\section{Susana Finquelievich}

Arquitecta. Master en Urbanismo por la Université Paris VIII. Doctora en Sociología Urbana por la École des Hautes Études en Sciences Sociales, París. Investigadora principal del Consejo Nacional de Investigaciones Científicas y Tecnológicas (CONICET). Co-directora del Programa de Investigaciones sobre la Sociedad de la Información y directora del Equipo I-Polis, Instituto de Investigaciones Gino Germani, Facultad de Ciencias Sociales, Universidad de Buenos Aires. (IIGG - UBA CONICET). sfinquel@gmail.com

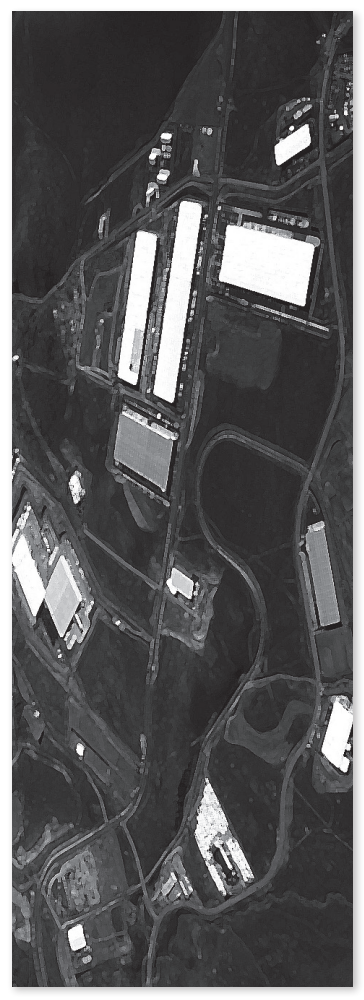

http://dx.doi.org/10.30972/crn.31315784 


\section{Ciudad aumentada y pandemia El habitar en el Orden Digital}

\section{Resumen}

Este artículo plantea interrogantes en torno a la ciudad como idea, espacio y sentido disciplinar. Reflexiona acerca de las relaciones entre lo urbano, la pandemia de la COVID-19 y su proceso de digitalización devenido de los sucesivos aislamientos preventivos, donde el Orden Digital se habría expresado como territorio emergente de procesos sociales, productivos y políticos. Busca promover así un debate disciplinar para la Arquitectura y el Urbanismo acerca de estos procesos. ¿De qué manera estas disciplinas se posicionan y caracterizan el fenómeno? ¿Cómo incide "lo digital" en las lógicas urbanas de conformación y prefiguración centro-periferia? ¿Qué nuevos territorios promueve la digitalidad en lo urbano, tanto en sus espacios públicos como privados? ¿Cuánto puede contribuir en este proceso epistémico la transdisciplinariedad?

\section{Palabras clave}

COVID-19; ciudad; orden digital; TIC.

\section{Augmented city and pandemic Living in the Digital Order}

\begin{abstract}
This paper raises questions around the city as an idea, space and disciplinary sense. It reflects on the relations between the urban, the COVID-19 pandemic and its digitization process from successive preventive isolations, where the Digital Order would have expressed itself as an emerging territory of social, productive and political processes. It seeks to promote a disciplinary debate for Architecture and Urbanism about these processes. How do these disciplines position and characterize the phenomenon? How does "the digital" affect urban logics of central-periphery shaping and prefiguration. What new territories does digitality promote in urban areas, both in its public and private spaces? Up to which point can transdisciplinary contribute to this epistemic process?
\end{abstract}

\section{Keywords}

COVID-19; Town; Digital Order; ICT.

\section{Cidade aumentada e pandemia O habitar na Ordem Digital}

\section{Resumo}

Este artigo suscita questões sobre a cidade como ideia, espaço e sentido disciplinar e reflete sobre as relações entre o urbano, a pandemia da COVID-19 e seu processo de digitalização resultante das sucessivas quarentenas preventivas, onde a Ordem Digital teria se expressado como um território emergente de processos sociais, produtivos e políticos. Procura promover um debate disciplinar para a Arquitetura e o Urbanismo sobre estes processos. Como é que estas disciplinas se posicionam e caracterizam o fenômeno? Como o "digital" afeta a lógica urbana de conformação e pré-configuração do centro-periferia? Quais são os novos territórios que a digitalidade promove nas áreas urbanas, tanto em seus espaços públicos como privados? Quanto pode neste processo epistêmico contribuir a transdisciplinaridade?

Palavras chave

COVID-19; cidade; Ordem Digital; TIC. 


\section{Tecnologías, ¿esta vez sí?}

Desde hace tres décadas, el paradigma informacional y su consiguiente revolución comunicacional inciden en lo urbano, transformando ciudades y territorios en sus dimensiones físico-espaciales y socioculturales. Este ensayo presenta discusiones en torno a estos procesos desde la reflexión contingente de esta pandemia. Problematiza las vinculaciones y relaciones emergentes entre lo urbano, la pandemia de la COVID-19 y el súbito proceso de digitalización devenido de los sucesivos aislamientos preventivos, donde "lo digital" se habría expresado como el territorio resultante (y habilitado) de procesos sociales, productivos y políticos. Se busca aportar así, a los campos disciplinares de la Arquitectura, el Urbanismo y la Sociología Urbana, inquietudes y preguntas que contribuyan al debate ontológico por comprender estas dinámicas y transformaciones socio-urbanas derivado de lo digital como proceso, sentido y espacio.

Esta contingencia sanitaria impone desafíos epistémicos y disciplinares: cómo caracterizar los procesos emergentes del hábitat urbano consecuente con una compulsiva digitalidad. Podemos cuestionarnos acerca de si la pandemia originó nuevos fenómenos en la ciudad o si profundizó los ya existentes. Ciertamente, la COVID-19 actuó como catalizadora de dinámicas presentes en los últimos años, vinculadas, en gran medida, con los avances tecnológicos digitales. Este trabajo retoma estas inquietudes y las articula a partir de discusiones conceptuales que recogen tres aproximaciones vinculadas con lo urbano en este contexto sanitario: la idea de ciudad, la experiencia social de sus espacios públicos y las incidencias de nuevos modos de vida en lo doméstico.

\section{¿Un ecosistema inocuo?}

Existe consenso (Finquelievich, 2016; Scolari, 2013; Rainie \& Wellman, 2012; Castells, 2006) acerca de que Internet se expresa como instrumento, herramienta y sustancia de lo digital. Visiblemente presente en la vida cotidiana, marca con sus avances y mutaciones procesos socioproductivos, culturales, económicos, territoriales y geopolíticos, ineludibles para comprender las actuales formas emergentes de lo urbano. La pandemia de la COVID-19 hizo evidentes estas nuevas configuraciones. No obstante, internet es solo una parte (fundamental) del llamado Ecosistema Digital (Katz, 2015; Tsatsou \& Elaluf-CaLDERWOOD, 2007; LÓPEZ GARCíA, 2005), concepto ampliamente utilizado para caracterizar las TIC $^{1}$ como entorno y relación socio-productiva. Según los autores, remite a una organización de componentes (artefactos, discursos, personas, datos e infraestructuras) que se
1. Tecnologías de la Información y la Comunicación. 
2. Cortez Oviedo, P. S. (2020).

Espacios públicos en el Orden Digital. El rol de las TIC en sus usos y apropiaciones. Editorial UNC; FINQUELIEVICH, S. (2016). I-Polis. Ciudades en la era de Internet. Diseño.

3. Con relación a la diferencia entre tecnología y técnica, entre el instrumento y la cultura que lo posibilita. El mito de la máquina (MUMFORD,

2017). desarrolla con equilibrios dinámicos y jerarquías tróficas. Es decir, representa "posiciones evolutivas dadas" de los elementos de la cadena de intercambio (digital).

Sobre la base de investigaciones anteriores ${ }^{2}$, este trabajo complejiza lo digital más allá de su idea de ecosistema, lo caracteriza como una semiósfera. Esto quiere decir que sus elementos no se disponen desde un sentido preestablecido (como lo indicaría un ecosistema), sino que expresan estructuras de poder, discursivas, y, por lo tanto, intencionadas. Se infiere que esta intencionalidad (geo-cultural) expresa la complejidad de lo digital como elemento significante de las actuales prácticas sociales. En consecuencia, inferimos que la denominación de Ecosistema Digital allanaría las tensiones discursivas que reflejan las contradicciones, desigualdades y expulsiones de lo digital como proceso sociocultural y político.

Este trabajo caracteriza lo digital como una semiósfera (LoTMAN, 2019), cuyos sentidos y dinámicas geo-culturales se encuentran encapsuladas, visiblemente a-localizadas y globalizadas. Representa una red de esferas de cosas, significados y flujos, cuya interacción denominamos Orden Digital (CoRTez OviEDo, 2020). Desde esta caracterización, lo digital remite a un orden socio-tecno-cultural capaz de incidir en las dinámicas socioproductivas, los procesos culturales y de consumo y, por ende, en la (re)producción del hábitat de lo urbano.

\section{El orden invisible}

La categoría de Orden Digital explícita una tecno-cultura que excede las tecnologías y técni$\mathrm{cas}^{3}$, y que abarca, fundamentalmente, sus discursos e intercambios culturales. Si comprendemos la cultura como trama semiótica y simbólica (GEERTZ, 2003), lo digital adquiere otra relevancia y perspectiva para (re)pensar la ciudad en este contexto de pandemia.

El Orden Digital se instrumenta socialmente por sus infraestructuras - Smartphones y redes de conectividad, Tecnologías Inmersivas y entornos ciberfísicos, Internet de las Cosas (IoT) y Blockchain, Inteligencia Artificial (IA) y algoritmos-, las que se integran en la experiencia de la vida cotidiana de las personas, inciden en sus experiencias y (con)vivencias en la ciudad, la geografía, las relaciones humanas y socioproductivas. Se infiere, además, por su simultaneidad, que el Orden Digital se expresa también por construcciones dialécticas y culturales: tráfico, consumo e intercambio de imaginarios y representaciones sociales, subjetividades e identidades sociales y políticas, en la forma de fotografías, videos, consumos culturales, streaming, posteos y debates públicos, y todo tipo de práctica social que medie lo digital. 
El Orden Digital expresa un nuevo territorio en los comienzos de la cuarta revolución tecnológica. Conforma una espacialidad emergente entre lo material y lo intangible. Es una hibridación del espacio-tiempo. Aquí radica uno de los nodos epistémicos - ¿y ontológico?-que la Arquitectura y el Urbanismo deberán afrontar en su comprensión fenomenológica. ¿Bajo qué presupuestos y saberes deberá aproximarse? Aún se acude a "lo virtual y lo real" como expresiones y categorías que reflejan la dinámica digital, ¿sigue siendo esto adecuado? En las últimas décadas, la revolución comunicacional también transformó los modos en que los humanos se comunican con los diversos dispositivos digitales y, entre sí, a través de ellos. Desde las antiguas conexiones dial-up a las actuales 5G, la convergencia tecnológica de los dispositivos hiperconectados con los que nos relacionamos, sumada a la plataformización de servicios y relaciones humanas, hace difícil identificar los límites concretos entre estas dos dimensiones.

Somos habitantes de una realidad expandida: (co)existimos en una hiperrealidad digital (BAUDRILLARD, 2002). Humanos y máquinas se relacionan reconfigurando una realidad múltiple conformada por archipiélagos de interacciones tecno-culturales. Al utilizar artefactos digitales nuestra vida cotidiana se expande, reconfigurando y ampliando redes y flujos de intercambios a-localizados en territorios efímeros y materiales simultáneamente, distantes en kilómetros, cercanos en bits y unificados por el uso y la apropiación social de la tecnología. Es el Orden Digital en su plena magnitud. No interesa dónde ni cuándo: mientras exista acceso digital habitaremos y circularemos los flujos de un nuevo habitar.

Este proceso evoca el surgimiento y transformación de la ciudad, donde las innovaciones tecnológicas actuaron como determinantes de la organización social y espacial, y a la vez condicionadas por las diversas necesidades y características de las estructuras sociales y del territorio. Las tecnologías que significaron una ruptura con el "modo de hacer" técnico y social preexistente ejercieron efectos revulsivos y duraderos, no solo sobre la estructura social, económica y espacial en la que se utilizaban, sino también sobre las ideologías y los modos de pensar. La misma estructuración de la forma "ciudad" y de sus diferentes conformaciones, a lo largo de la historia humana, es la traducción en el espacio de la incorporación de mutaciones técnicas en las formas de hacer y de organizar. Basta recordar la multiplicidad de ciudades fabriles que surgieron de la revolución industrial, las ciudades satélites posibilitadas por el auge de los automóviles, los transportes públicos y el teléfono, el crecimiento de las megalópolis, y como producto de la década de los 80, del avance de la ciencia y de la tecnología volcadas a la productividad, lo que se percibía entonces como una forma emergente de conformación urbana: las Tecnópolis. 
Las tecnologías y los sentidos que las instrumentan no son inocuas para el habitar urbano. Como se expresó al principio, la COVID-19 catalizó procesos latentes que ya incidían en la ciudad. Desde esta mirada, el Orden Digital queda expuesto como emergente espacial y sociocultural del paradigma informacional, que previamente a esta contingencia sanitaria ya planteaba inquietudes y la necesidad de otras preguntas y nuevas categorías. Esta pandemia lo ha develado en sus particulares formas de vivenciar lo urbano. ¿En qué ciudad habitamos al estar recluidos en nuestros hogares? ¿Cómo son y de qué manera se manifiestan nuestros intercambios sociales en este contexto digital y pandémico? ¿Cuáles son nuestros lugares de trabajo, recreación y ocio: la casa, el ciberespacio, los pulsos eléctricos?, ¿o más bien enajenados archipiélagos de experiencias y consumos? ¿Todos y todas somos parte de la hiperrealidad del Orden Digital? ¿Cómo se expresan las nuevas segregaciones urbanas? Son algunos de los interrogantes de partida que serán abordados en el próximo apartado y que constituyen el núcleo discursivo del trabajo.

\section{Una mirada a escala}

El trabajo desarrolla un análisis crítico desde un enfoque interpretativo y exploratorio, cuyo aporte principal es el teórico-conceptual. El ensayo emplea una metodología multimodal de triangulación de autores y ejemplos enunciativos articulados por una escritura dialéctica con foco en el interrogante. Como consecuencia de las limitaciones debidas a la pandemia de la COVID-19, el trabajo de campo fue reducido. Esto se suplió recurriendo a fuentes secundarias referidas al problema (reseñas periodísticas, informes audiovisuales y de plataformas e informes técnicos parciales), que se contrastaron con el análisis reflexivo de las notas de campo de investigaciones previas (con relación a los espacios públicos y sus consumos culturales, el turismo y el trabajo en la economía digital, en los contextos de Córdoba, Buenos Aires y Santiago de Chile).

La estructura discursiva desarrolla el problema con relación a lo urbano, los espacios públicos y lo doméstico. Este apartado articula tres puntos que recuperan escalas de aproximación:

- 1:10000 | Ciudad del aire: expone una caracterización de lo urbano desde la perspectiva digital en el contexto de pandemia;

-1:1000 | Nuevos vínculos y espacios: se enfoca en los fenómenos emergentes de los intercambios socioculturales devenidos de la interacción entre lo digital, la ciudad y la COVID-19 $\mathrm{y}$

- 1:100 | Habitar los flujos: refiere a los sentidos, mutaciones y expresiones del habitar doméstico consecuente del aislamiento. 


\subsection{1:10000 | Ciudad del aire}

$\mathrm{Al}$ abordar las relaciones entre lo urbano y las tecnologías, el concepto de Smart Cities surge predominante entre otros emergentes del Orden Digital. No obstante, esto refleja solo una parte, importante, de las aplicabilidades de lo digital en las ciudades, pero no profundiza en aclarar sus dinámicas socioterritoriales, las que interesa indagar. El pensamiento de SiLva (2016) aporta los primeros andamios en esta escala de aproximación. Plantea una primera relación acerca de lo urbano y lo digital: la ciudad imaginada — con relación a su obra Imaginarios Urbanos (2006) - y la ciudad digital van de la mano, ambas son etéreas y asociadas, una es mental y la otra, del aire. Esta metáfora es un potente imaginario que representa las nuevas territorialidades urbanas devenidas de lo digital en el contexto COVID-19: efímeras, simultáneas, pero al mismo tiempo, concretas en las transformaciones de las prácticas sociales y físico-espaciales de la ciudad tradicional (restringida en sus dinámicas corporales en este escenario sanitario).

En los años 60, empezó a difundirse la idea de que la ciudad moderna era algo más que un conjunto de edificios y calles. La ciudad comenzó a ser percibida como un inmenso nodo de comunicaciones, un entramado caótico de mensajes, almacenamiento, transporte, una máquina compleja que incluía los edificios, pero también el mass-media. El grupo Archigram, enmarcado en el antidiseño, era futurista, antiheroico y pro-consumista. Soñaban con realizar una ciudad que se construía a sí misma de manera imprevisible, cibernética, con edificios inflables, células habitacionales (transferibles y repetibles), proyecciones y rayos láser. Sus obras ofrecían visiones seductoras de una ciudad glamorosa en la era futura de las máquinas (Finquelievich, 2016).

También en esa década se abrió una puerta hacia el ciberespacio, un territorio que necesita ser planificado y organizado constantemente. No en vano se habla de la arquitectura del ciberespacio. PIERRE LÉVy (2004) propone que los arquitectos del ciberespacio pueden no proceder de los círculos del arte tradicional, como ingenieros, diseñadores de redes o de interfases, creadores de programas, juristas de la información, etc., que van a estructurar en gran medida las evoluciones sociales y culturales. Por su parte, BENEDIKT (2020) predice que los arquitectos del ciberespacio, educados en programación y en ciencias informáticas, en gráficos y en diseños abstractos, diseñarán junto con sus colegas, los arquitectos del "espacio real”, edificios electrónicos que serán tan complejos, funcionales, únicos, envolventes y hermosos, como sus contrapartes físicas. 
4. Con relación a la dialéctica estructura-agencia planteada por BOURDIEU desde su noción de habitus, entendiéndose la ciudad como actante no humano que se transforma, se proyecta y se agencia producto de sus relaciones e interdependencias con la técnica y sus tecnologías.

\section{Arqueología de lo urbano}

Continuando con el andamiaje de lo urbano, SenNett (2019) propone recuperar la diferenciación entre ville y cité, entre la totalidad y la parte. Su planteo describe una distinción básica: el entorno construido es una cosa, cómo las personas lo habitan es otra. Es consecuente con su pensamiento acerca de los conceptos romanos de Urbs y Civitas, la relación entre el componente físico-espacial y sociocultural y político de una ciudad. Esto dialoga con lo que FERNÁNDEZ (2014) propone como sito-geografía, pensar lo urbano desde la particularidad de la experiencia situada. Ambos autores coinciden en que la ciudad no es un componente único ni totalitario, sino el ensamble de variadas dimensiones y relaciones, materiales e intangibles, simultáneas y tecnológicas.

La Arquitectura, y el Urbanismo en particular, como fruto de su antigua tradición funcionalista, segmentaron los estudios urbanos centrándose en la dimensión físico-espacial de lo urbano. Esto resultó en una amplia comprensión de la materia: entender la ciudad como conjuntos de infraestructuras físicas, palpables, habitables y ambientales, pero se relegaron sus componentes sensibles asociados al aura social de esos elementos (Benjamin, 2003). Dicho de otro modo, simplificó lo urbano a una sola de sus expresiones: la euclidiana. Con un sentido crítico, HéNAFF alude a esta discusión con estas palabras:

Tal fenómeno dio lugar a una situación extraña: el éxito mundial de la forma urbana parece confundirse con la derrota de la ciudad o, por lo menos, con la de los que ha sido deseado y construido durante milenios a partir de la idea de ciudad (2016, p. 48).

A partir de este planteo, se puede consensuar que la idea de ciudad mutó con los siglos. Estos autores coinciden en que se trata de un proceso social con expresión física y espacial, que excede su forma urbana (Morris, 2018). La ciudad, como fenómeno de lo urbano, refiere a un concepto en tensión (SolÀ-Morales, 2006; Ascher, 2004), particularmente agenciada ${ }^{4}$ por los desarrollos de la técnica y las prácticas sociales que promueve y usufructúa; singularmente afectada hoy por la pandemia de la COVID-19. Se puede cuestionar si son la técnica y sus tecnologías las que modifican procesos culturales que dan forma a lo urbano o viceversa, pero no puede negarse su interrelación. Es así que lo urbano está siendo transformado, desde su experiencia, por el Orden Digital, tanto en sus dimensiones urbs como civitas.

Fue Echeverría, en "Telépolis", quien introdujo uno de los conceptos emergentes respecto de lo urbano a inicios del paradigma informacional en los años 90, y que hoy adquieren nueva resonancia. Telépolis es la metáfora de la ciudad remota, distante, pero integrada 
desde la comunicación. "Telépolis ha surgido a partir de las metrópolis y de la expansión del capitalismo avanzado, y en particular gracias al desarrollo exponencial de los mercados de la información y de las comunicaciones” (EcheverRía, 1994, p. 27).

La ciudad del aire (global y local) que describe el autor se vincula directamente con lo propuesto por CARRIón (2020), quien desde la contingencia sanitaria y el aumento de la penetración y el tráfico digital propone repensar lo urbano desde el concepto de Teleciudad. Manifiesta que lo digital genera y promueve nuevas espacialidades que inciden en lo urbano alterando su concepción y forma. Desde esta reflexión señala tres tipologías de abordaje al fenómeno: la relocalización de la dimensión física-espacial a la intangible; la deslocalización vinculada con la dinámica centro-periferia-centro y la alocación de relaciones socio-urbanas asociadas a los servicios globales de la economía de plataformas. En su pensamiento, hemos pasado de una urbanidad de lugares a una de flujos -en alusión a los espacios de flujo propuestos por CASTELLS (2006)—.

\section{¿Ciudades post-humanas?}

A partir de estas discusiones, surgen reflexiones y prospecciones acerca de lo urbano y su sostenido proceso de mediatización. La metáfora de la ciudad del aire de SiLva representa un imaginario social potente que pone en sentido las ideas de SENNETT, ECHEVERRía, LÉvy y BENEDIкт. La ciudad como fenómeno urbano no es ya un territorio uniforme y, menos, uno formal, sino relaciones sociales espacializadas en red y también extendidas de modo rizomático y glocal, producto de la injerencia de bits y flujos. HéNAFF propone repensar este proceso asumiendo, críticamente, el declive histórico de las ciudades. Desde su sentido, se asume la derrota del urbanismo físico-espacial. Es decir, existe para él una tensión no resuelta entre el "éxito" del proceso de urbanización global —urbs/ville - y su fracaso como forma y relación social - civitas/cité-. Esto encuentra relación estrecha con el pensamiento de BoRjA y CASTELLS: "la globalización, la informalización y la difusión urbana generalizada parecen converger hacia la desaparición de la ciudad como forma específica entre territorio y sociedad” (2006, p. 12). En estas dinámicas y procesos que afectan la ciudad, no solo como idea, sino lugar y espacio, ¿qué rol juega el Orden Digital?

Hasta hace poco, cuando el mundo era más lento, los edificios se correspondían con las instituciones, las significaban, las hacían visibles. La organización interna de los edificios, los sistemas de circulación, las jerarquías de espacios de privacidad y control reflejaban -aún reflejan- la estructura de la institución y diagramaban físicamente un modelo de 
actividades. Existía una morfología y un sistema de símbolos arquitectónicos propios de los hospitales, una diferente para los bancos, otra para las escuelas, y así con respecto a todos los inmuebles y programas. La función y la forma se correspondían, y si había una no-correspondencia entre ellas (un edificio podía, por ejemplo, quedar chico para sus funciones), el edificio debía modificarse (Finquelievich, 2016). Todo esto mutó en la última década, como producto del Orden Digital, y se ha acelerado en esta contingencia sanitaria.

Actualmente el software supera al hardware. En vez de construir nuevas alas a los edificios, se implementan sistemas que almacenan la información. Las grandes bibliotecas universitarias están en línea.

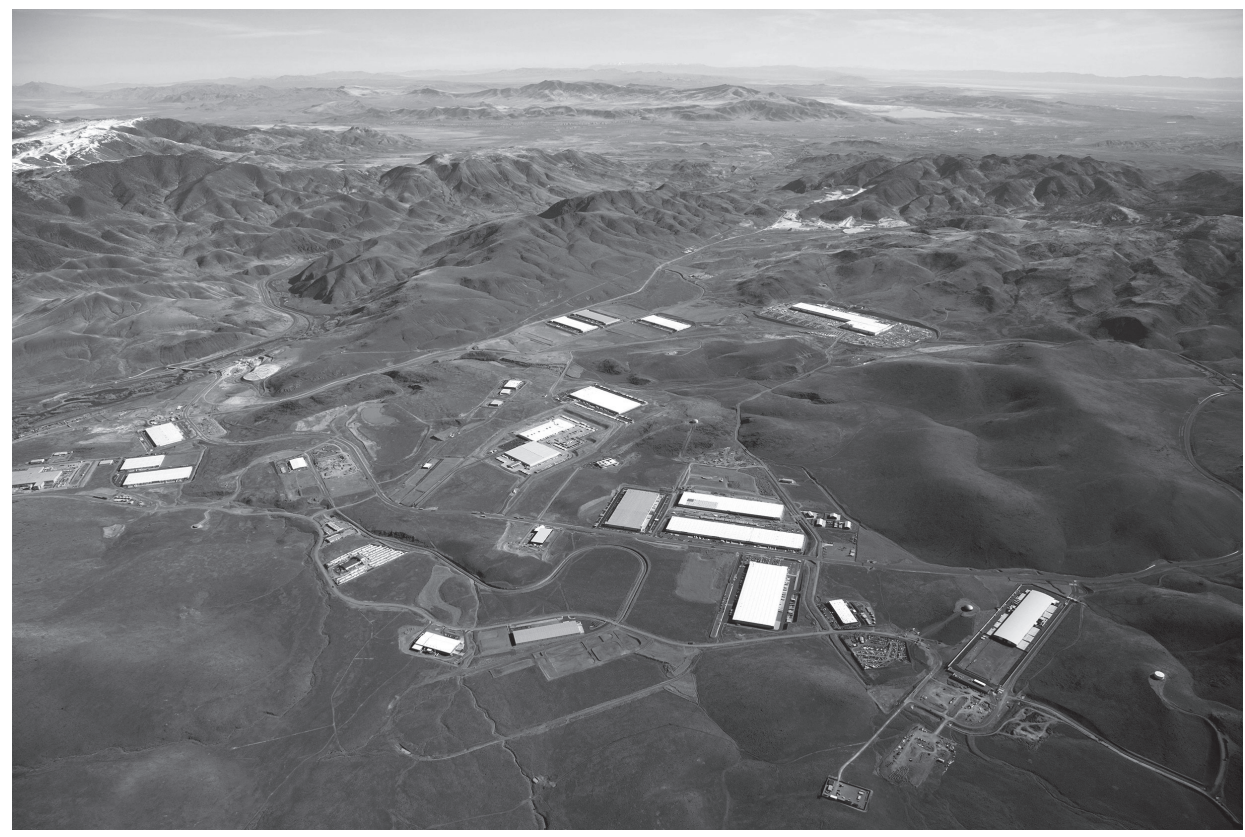

Figura 1. ¿Ciudad sin habitantes? Tahoe Reno Industrial Center en el desierto de Nevada (USA), sede de los conglomerados tecno-digitales de Google. Apple y Tesla y uno de los data center globales núcleo. Evan Petty, Guggenheim.org (2020) 
El diseño de bibliotecas, bancos y algunas instituciones públicas ha sido redefinido. No se trata ya de proyectar un edificio con depósitos de libros y áreas de circulación, capaz de albergar los kilómetros de estantes necesarios para guardar las colecciones, sino de diseñar y programar las herramientas informáticas para almacenar, buscar, encontrar y mostrar los textos digitalizados. Por lo tanto, la biblioteca, los bancos, los edificios administrativos, las empresas, son extensibles y reconfigurables en softwares, no en ladrillos. Procesos similares se están dando en universidades y centros de estudios, donde cursos y carreras virtuales o semivirtuales reemplazan las ampliaciones físicas de los campus (Finquelievich, 2016).

En relación con esto, Rem KoolhaAs ensaya y propone un nuevo pensamiento de ciudad: lo denomina arquitectura post-humana. Un fenómeno urbano que se expresa relocalizado, deslocalizado y a-localizado de manera simultánea, y que integra la ciudad histórica (sociedad-territorio) y lo rural con los flujos de datos y servicios (sociedad-procesos productivos). Un concepto urbano plasmado en su obra Countryside, The Future (2020). Una ciudad del aire cuyos componentes tangibles se expresan por edificios cuyos programas genéricos albergan instalaciones e infraestructuras, procesadores y algoritmos, especializándola (en parte). Ejemplo de ello son los crecientes núcleos tecnológicos en el "norte global", como Tahoe Reno Industrial Center en el desierto de Nevada. Se trata de la sede física de uno de los data-center que posibilitan nuestro teletrabajo, los intercambios sociales en la web y los consumos culturales 4.0 que abastecen el entretenimiento diario. A pesar de la distancia en kilómetros con este sitio, nos une a él la interdependencia del Orden Digital. Según ReM KoolHAas, la ciudad histórica probablemente cambie más por lo que sucede fuera de ellas que por lo que ocurre dentro (Wainwright, 2020).

Para concluir este primer apartado acerca de la ciudad como fenómeno contextual, se evidencia la injerencia del Orden Digital. Como remarcamos en párrafos arriba, la COVID-19 no implicó per se transformaciones en lo urbano, al menos desde esta escala de aproximación. No obstante, es cierto que la coyuntura sanitaria hizo visibles y aceleró procesos latentes que ya se gestaban; por ejemplo, aquellos relacionados con dinámicas de difusión centro-periferia y el crecimiento urbano por expansión y densificación concentrada. Si se pretende pensar la ciudad como idea (a partir de la pandemia), sería un error considerarlos propios de esta contingencia.

Es pertinente aclarar que, dada la poca perspectiva histórica y los estudios al respecto, es prematuro aseverar de manera cuantitativa cuánto la pandemia potenció de por sí los 
5. Fenómeno asociado a procesos de difusión regional/urbana y redistribución interna de población, resultantes de percibir "la ciudad" como entorno poco saludable y nocivo en el marco de una lógica neo-higienista. En el contexto de la provincia de Buenos Aires, se escribe: "Desde el inicio de la pandemia se multiplicaron por 10 las consultas que recibió una fundación (Es Vicis, Fundación para el repoblamiento rural) para migrar a pueblos. El encierro y la posibilidad de teletrabajar aceleraron la tendencia a buscar entornos más relajados, con menos viajes $y$ cerca de la naturaleza. Quienes dieron el salto temían problemas de conectividad o el riesgo de estancamiento profesional, pero hoy notan que pueden desarrollarse lejos de los grandes centros urbanos”. En cuanto a la experiencia de los vecinos, se agrega: "Se hizo evidente que no necesitamos la oficina para seguir haciendo nuestro trabajo". Tuchin, F. (1 de octubre de 2020). Del "delirio" a la tranquilidad: por qué cada vez más personas cambian las grandes ciudades por pequeñas localidades. Redacción. https://www. redaccion.com.ar/del-delirio-a-la-tranquilidadpor-que-cada-vez-mas-personas-cambian-lasgrandes-ciudades-por-pequenas-localidades/ Para el caso de Villa Gesell, se comenta: "En abril esta tendencia se consolida: más de 15.000 personas ingresan a la ciudad y un cuarenta por ciento solicita estadías por más de un mes, mientras un quince por ciento lo hace para el resto del año" Chaina, P. (mayo de 2021). Villa Gesell y la mudanza en pandemia. Página12. https://www.pagina12.com.ar/343568-villagesell-y-la-mudanza-en-pandemia. En síntesis, por el momento no puede decirse que esta es una tendencia estadísticamente significativa, dado que por la novedad del fenómeno no han podido realizarse estudios a fondo, pero marca una corriente que merece ser analizada. procesos de expansión urbana en poblaciones rururbanas -aunque existen antecedentes asociados que dan cuenta de aumentos de población en ciudades medianas y pequeñas en Argentina durante 2021_ ${ }^{5}$. Aun así, se puede reconocer que la COVID-19 renovó ciertos imaginarios sociales y comunicacionales asociados a este posible fenómeno de reconfiguración físico-espacial de lo urbano: una "mejor calidad de vida" catalizada hoy por discursos neo-higienistas que refieren a "los entornos naturales", el "aire puro", la distinción de "la libertad" y una "mayor seguridad". Ciertamente, estas dialécticas comunicacionales, varias de ellas potenciadas por las TIC, encuentran un contexto propicio en la relocalización, la deslocalización y la alocación de procesos productivos y sociales devenidos del teletrabajo, la educación a distancia, el e-comercio y la telemediación de relaciones sociales resultantes de la pandemia.

Sí podemos plantear como fenómeno propio de esta contingencia las desigualdades expuestas de la ciudad del aire, en tanto acceso, pertenencia y desarrollo de sus habitantes. Apagado o disminuido el mundo análogo, aquellos procesos y personas que mejor vinculados estaban con lo digital tuvieron un menor impacto en términos económicos y socioculturales. Por el contrario, las y los expulsados del Orden Digital padecieron las asimetrías de desarrollo y habilidades que impone la brecha tecno-cultural. En otras palabras, surgieron nuevos segregados/as urbanos. ¿Cómo los incorporamos? ¿Es solo una cuestión de acceso digital, o se precisan otras lecturas atingentes?

El Orden Digital configura un nuevo territorio, una ciudad aumentada, cuyos componentes materiales e intangibles complejizan y transforman las problemáticas urbanas y sociales de la ciudad histórica, a la vez que agregan otras emergentes. Los nuevos flujos, las experiencias e intercambios culturales y socio-físico-productivos que promueve lo digital representan un desafío disciplinar actual. En esta nueva arquitectura de lo urbano, adelantada por la pandemia, se generan y promueven nuevos espacios y lugares, otros vínculos y relaciones y otros modos de (con)vivir. ¿De qué manera y cómo se abordará esta urbanidad emergente? ¿Qué nuevos desafíos disciplinares y epistémicos plantea? 


\section{$2.21: 1000$ | Nuevos vínculos y espacios}

La COVID-19 transformó (¿de manera transitoria?) la humanidad ante la incertidumbre de lo desconocido. Las ciudades se vaciaron y enmudecieron, y sus habitantes se aislaron en sucesivos e intermitentes confinamientos. De manera diferencial, el mundo análogo se detuvo en Argentina durante el período $2020^{6}$ quedando en funcionamiento aquellas actividades esenciales, mientras que el resto del espectro socioproductivo dependió de su grado de digitalización (previa). Estados y administraciones gubernamentales, grandes y pequeñas empresas, economías emergentes y convencionales, escuelas e instituciones y hasta las propias relaciones sociales se transformaron.

Desde entonces, y producto de las restricciones físicas-espaciales, el coronavirus limita distintas dimensiones de lo urbano a la intangibilidad. Esta contingencia produjo, y consolida, un éxodo acelerado de lo análogo a lo digital en diversos intercambios: estatales, comerciales, laborales, sociales y educativos. Las restricciones físicas obligaron a reconvertir sus sentidos, y en ese proceso se agudizaron desigualdades prexistentes. Esto evidenció la faceta social del Orden Digital. En tres décadas de revolución comunicacional, nunca antes la brecha tecno-cultural de lo digital había sido tan decisiva para el trabajo, la economía y los vínculos sociales de las personas. Esto expresó cuán profundas son las transformaciones de lo digital en el hábitat urbano; entonces, asumiendo esta injerencia, cabe preguntarnos: ¿se puede disociar el derecho a la conectividad de un nuevo derecho a la ciudad?

\section{Una historia prospectiva}

Retomando la perspectiva histórica, las correspondencias entre ciudad, tecnología y sociedad no son novedosas. Con claroscuros, la técnica se hizo presente modelando lo urbano y su forma social. Esto se verifica en las incidencias que tuvieron las tecnologías de los caminos, puentes y acueductos romanos, las tecnologías del vapor, los ferrocarriles y telégrafos en la integración de territorios geográficos que estructuraron figuras administrativas como el Estado-nación. En lo social, las tecnologías también incidieron en procesos culturales y dinámicas políticas promoviendo nuevas formas y difusión del conocimiento, la participación y construcción de ciudadanía, las que gestaron revoluciones y transformaciones sociales. Con esta mirada, ¿qué nos hace pensar que el Orden Digital dejaría de promover cambios similares?

En los 60, la convergencia y la sinergia de un conjunto de innovaciones técnicas abrieron una fase clave en el proceso de urbanización reciente, particularmente en Europa, que se derramaría poco después sobre las ciudades de otros continentes. Entre estas innovaciones, las más
6. Según el Tablero de Impacto del Coronavirus del BID, la movilidad de las personas en Argentina se redujo con respecto a febrero de 2020 un $45 \%$ en promedio durante el período marzojulio, con un pico de $68 \%$ en el inicio; mientras que para el período agosto-diciembre esta caída se redujo al $25 \%$, según el estudio realizado por Grandata Lab. Ambas mediciones se basaron en la misma metodología: el análisis de datos de desplazamientos de celulares (no geolocalizados) a partir de la triangulación con antenas de telefonía móvil. 
7. Con relación al planteo de KoOLHAAS, y con sentido en la discusión de la descorporización urbana. determinantes conciernen a los transportes y la comunicación: acortar distancias era la meta. Las redes de transporte del tren (TGV) y del metro a gran velocidad; los grandes cargueros que multiplican la velocidad y la capacidad de las redes aéreas; las nuevas aplicaciones del teléfono con la consulta a distancia de datos informatizados y la transmisión inmediata de mensajes escritos: todos estos instrumentos confieren a sus usuarios cierto grado de ubicuidad.

Esta nueva realidad parecía confirmar a primera vista lo que planteaba CLIFFord SIMAK en su libro de cuentos Ciudad: "La ciudad es un anacronismo. Se ha sobrevivido a sí misma" (1988, p. 14). En un principio, la ciudad era el lugar en que se agrupaban los miembros de una tribu para protegerse mutuamente. Luego se rodeó de una muralla para aumentar la protección. Posteriormente la muralla desapareció, pero la ciudad siguió viviendo a causa de las ventajas que ofrecía al tráfico y al comercio. Y llegó a nuestros días porque la gente se veía obligada a vivir cerca de sus lugares de trabajo, y los trabajos estaban en la ciudad. Pero todo esto ha cambiado, ha quedado expuesto por el simulacro del aislamiento global, ya que la pandemia hizo reales los escenarios más distópicos. Se produjo una limitada migración de clases medias y altas, desde la ciudad a barrios residenciales de la periferia 0 del campo, o a pueblos y ciudades más pequeñas. Se demostró que gran parte del funcionamiento urbano puede prescindir (momentáneamente) de los cuerpos en la ciudad. Por consiguiente, ¿es posible una ciudad deshumanizada? ${ }^{7}$ ¿Qué nuevas formas y tensiones de lo social emergen de esta dinámica?

\section{Ciudad enmudecida}

En un mundo experiencial más achicado que en los 60, la comunicación, el transporte y la necesidad de cierta instantaneidad parecen prevalecer. Así surgen nuevos vínculos y espacios, físicos y sociales, materiales e intangibles. La ciudad aumentada parece concretarse en las redes de intercambios del ciberespacio, articulada por autopistas de bits, data-center y fibras ópticas, segmentada y fragmentada por velocidades y accesos, y vivenciada desde la intermediación de las pantallas. Desde muchos sentidos, la pandemia nos restringió a esta ciudad del aire, glocal y ubicua, y habilitó nuevos espacios sociales y públicos. Como retrata la obra Cities of silence (AA. VV., 2020), la ausencia de los humanos en plazas y parques, calles y edificios públicos permite otra perspectiva de lo urbano.

Lo que parece la mejor escena para recorrer una ciudad o tomar una fotografía sin temor implica también el enmudecimiento de su sentido político. La nueva perspectiva de lo urbano que aportó el aislamiento puso en evidencia, aún más, el sentido (faltante) de sus 
espacios públicos como lugar de conflicto, disputa, identidad e intercambio social. La COVID-19 terminó por develar a la internet como soporte y reducto de estas interrelaciones socioculturales de lo público (HABERMAS, 2009), pero no sustituyó a la calle. Como ciudadanos de la ciudad aumentada, desarrollamos y apropiamos nuevos espacios públicos y simbólicos articulados por plataformas y redes sociales, posibilitados por aquellas instalaciones post-humanas que sugiere KoolHaAs.

Esto suscita interrogantes acerca de qué tipo de espacios públicos son estos, donde nuestros cuerpos se reducen al régimen escópico ${ }^{8}$ y a la performatividad mediatizada por avatares, consumos y tendencias en redes y cuentas trolls. ¿Qué tipo de sociedades promueve esta interconstrucción socio-política? Ejemplo de ello fue la proliferación de los movimientos terraplanistas en plena contingencia, cuyo fenómeno también es prexistente a la pandemia. Existe consenso de que este término nuclea los movimientos en redes sociales (YouTube, Telegram, WhatsApp, Facebook) asociados a las teorías de la conspiración, pero también a minorías emergentes radicalizadas en la búsqueda de visibilidad y (des)marcación. Durante la COVID-19 estos movimientos se reconfiguraron con nuevas luchas y sentidos: anticuarentenas, antivacunas, grupos ultras y radicales, etc., que a partir de constantes ataques mediáticos y desinformación promovieron la infodemia y las fake news que jaquearon la situación sanitaria de sus países y la representatividad democrática.

Esta intensidad del intercambio social en los espacios públicos de las redes coexistía en un principio con el silencio de las calles. Mientras la ciudad histórica enmudecía, la ciudad aumentada (del aire) experimentaba un desarrollo sin precedentes. En Argentina, el aumento del tráfico de internet en el período abril de 2019-abril de 2020 fue del $65 \%$, y del $35 \%$ más entre marzo-abril de 2020, cifra que se mantuvo en el segundo semestre con relación a igual período de 2019 (CABASE, 2020). Esta mayor interacción en las redes se tradujo en nuevos vínculos y espacios, pero también en nuevas violencias en esta urbanidad emergente. Según el INADI (2021), los casos por acoso, discriminación y hostigamiento aumentaron en 2020 un $65 \%$ con respecto a 2019, siendo la comunidad LGTTBIQ+ $(15,2 \%)$ y las mujeres (9 \%) los grupos más vulnerades/as. Se señala también que las tres plataformas que más registraron denuncias por violencia y discriminación fueron Facebook (45,2 \%), Instagram (14,9 \%) y Twitter (9.1\%). ¿Qué tipo de legislaciones necesita esta ciudad aumentada para garantizar la convivencia de sus espacios públicos?

\section{Refiere a la} interdependencia de las y los sujetos de mirar y se visto en las redes $y / 0$ fotografías (AUMONT, 2013; JAY, 2003), idea asociada al concepto de Pulsión Escópica en el psicoanálisis lacaniano. MasotTa (2018) plantea esta perspectiva como una evolución de la cultura visual. 


\section{Artículo de Fondo}

9. La República Argentina declaró como servicio público

estratégico la Internet, la telefonía móvil y la televisión

por cable en julio de 2020,

y promovió y generó una

Prestación Básica Universal

y Obligatoria $(\mathrm{PBU})$ que

garantiza datos (5GB),

servicio de telefonía móvil y

de televisión por cable a un costo asequible (DNU 690/20).
Desde la discursividad del trabajo, bien se puede caracterizar estas plataformas como espacios y lugares urbanos de una nueva y emergente ciudad, también opacos e iluminados como sugiere Milton Santos. Ciertamente, la pandemia de la COVID-19 pone blanco sobre negro en los procesos latentes de la ciudad física, los que también se expresan en la digitalidad. La ciudad aumentada se presenta compleja y simultánea en el Orden Digital, con las mismas violencias que subyacen y se ocultan en la ciudad histórica, con sus desigualdades y expulsiones. En este afán por acortar distancias urbanas, como lo evidenció el futurismo de los 60, actualmente no solo es necesario tender cables de fibra óptica y proveer mejores velocidades, sino facilitar el acceso digital en términos socioculturales, asumiendo que en última instancia se está garantizando el acceso a los espacios públicos emergentes. Esto se traduce en que ciertos Estados durante la pandemia hayan declarado la Internet y su accesibilidad como servicios públicos estratégicos ${ }^{9}$, iniciativas que remiten a la declaración del acceso a Internet como derecho humano por parte de la ONU (2016).
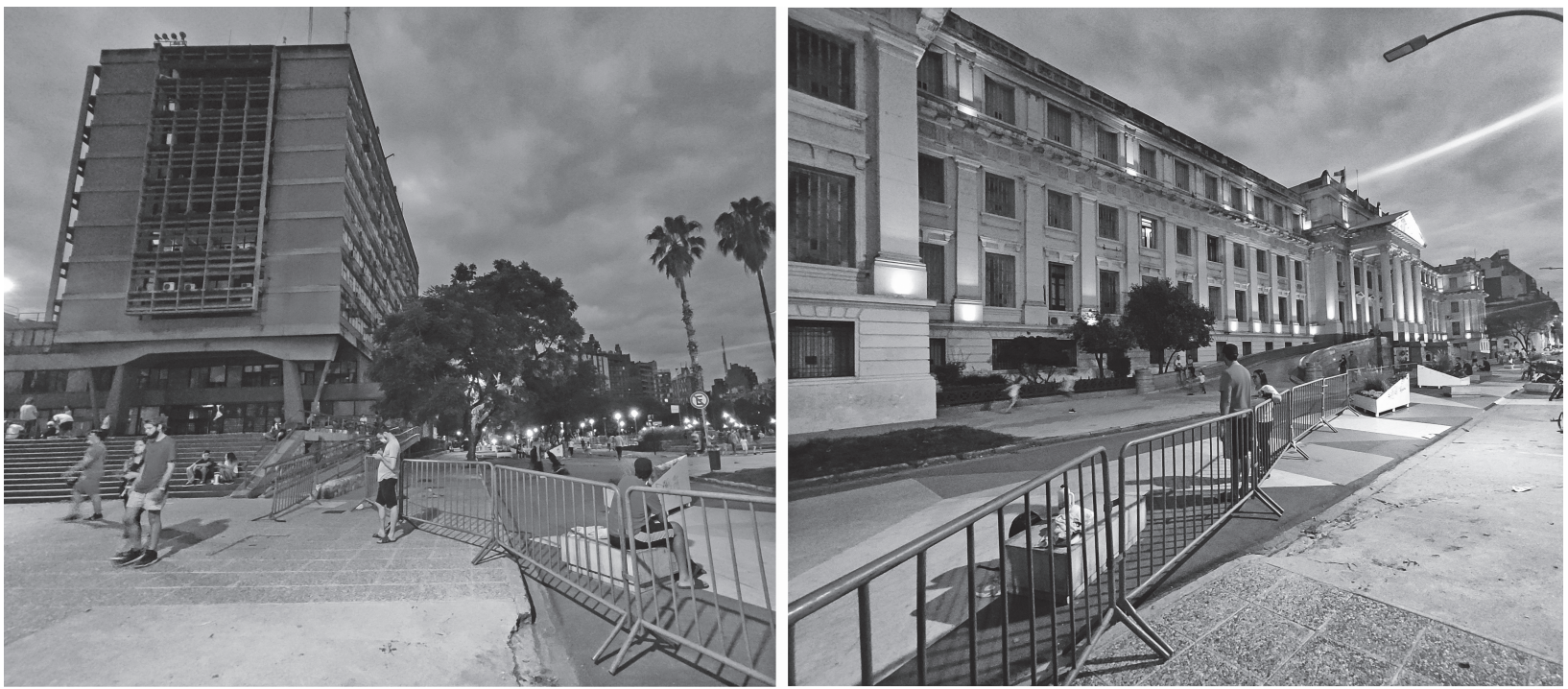

Figura 2. La ciudad vallada. Imágenes del centro de la ciudad Córdoba y áreas peatonales valladas durante el año 2020. Elaboración propia (2020) 
Existen diversos ejemplos de internet y las redes sociales como constructoras de nuevos vínculos y espacios sociales y políticos. Hay un hilo conductor desde la Primavera Árabe hasta la ocupación del Capitolio de los Estados Unidos: la cada vez mayor injerencia de las redes sociales en la ocupación de la calle. Ciertamente, es en este espacio y lugar donde se expresan las y los ciudadanos, no sin antes (re)configurarse en las redes digitales. Durante 2020, a pesar de los aislamientos, no se pudo prescindir de los cuerpos (políticos) en corredores, parques y monumentos de la ciudad histórica. A pesar de su enmudecimiento (intermitente), la calle sigue siendo el espacio de lo público como lugar que ocupar y disputar, y debe preservarse con este sentido. Han sido notorios con la excusa de la contingencia sanitaria los abusos de poder en los espacios públicos tradicionales: detenciones arbitrarias, pérdida de derechos y áreas céntricas y parques vallados que promovieron un imaginario social prohibitivo de la ciudad histórica y se replicaron en otros contextos urbanos como ejemplos "legitimados" del control social, posibilitados por el Orden Digital y su ubicuidad comunicacional. Estos procesos sí son propios de la pandemia de la COVID-19, por la escala (simbólica y digital) en que se ejercen y replican en los imaginarios sociales de la ciudad aumentada. Desde esta asociación, y en función de asegurar el derecho político en la ciudad física e intangible, ¿desde qué caracterización de espacio público garantizaremos su derecho y acceso?

\subsection{1:100 | Habitar los flujos}

En esta última escala de aproximación a los fenómenos de la ciudad aumentada, se encuentran aquellos vinculados con los cuerpos y la proximidad, la pequeña escala. Así como la ciudad se transfiguró producto del Orden Digital, también lo hicieron la casa y la vivienda, y se redefinió lo doméstico. Entre las ideas de Ryвczynski (2013) y las de Coll López (2018) existe una transformación evidente de este ámbito. Entre el conjunto de sensaciones y emociones percibidas en torno a la idea de hogar y el espacio extremamente flexible que se corresponde con tiempos líquidos que se diluye en el aire. Coll López plantea lo siguiente:

[C]uando nuevos modelos domésticos aparecen a partir de conceptos derivados de las comunas hippies de 1966, nuevos modos de habitar basados en nuevas relaciones sociales y familiares: del "espacio doméstico" se pasa al "paisaje doméstico", se rompe con las convenciones de una vivienda subdividida en habitaciones, amueblada. Cojines, muebles modulares, espacios fluidos, vivienda flexible son elementos y estrategias [...] de relaciones sociales más libres (2018, p. 66).

El autor denomina "mueble habitable" a esta nueva relación socio-espacial del ahora paisaje doméstico. Un habitar doméstico que no solo excede el ámbito propio de sus programas 
físicos y cuantitativos, sino que incorpora relaciones sociales más amplias, incluso por fuera del ámbito de la vivienda. En la última década se ha difundido el interés por recategorizar la vivienda desde sus programas o sus transformaciones. Y es que cada época se corresponde con un sujeto social, costumbres e interrelaciones, y estos con formas emergentes de habitar la ciudad y la casa. La expresión "nuevos modos de habitar” ha construido un imaginario fecundo que promueve estudios y reflexiones, fundamentalmente en la Arquitectura. Como expresa SARQuis (2011), debe existir una coincidencia entre el pensamiento proyectual y la gente. Sin embargo, aún son escasos los estudios que dan cuenta de lo doméstico como una relación social espacializada y, quizás, deslocalizada y alocalizada; más aún como resultante de una ciudad aumentada en un Orden Digital.

\section{La célula tangible}

La pandemia de la COVID-19 evidenció la vivienda como el lugar extremadamente flexible que plantea Coll López (2018), sin que esto deje de generar tensiones con la idea de hogar que considera RyвсZyNsкi (2013). Justamente, fue durante los confinamientos cuando la casa como espacio y la vivienda como lugar fueron el ámbito donde se hibridaron dos mundos: el análogo y el digital. La vivienda individual y, particularmente, la colectiva se vieron afectadas por los confinamientos. La obligatoriedad de permanecer en ellas generó usos y apropiaciones que no solo alteraron sus programas, sino que también evidenció su obsolescencia conceptual.

Los ámbitos privados se reconfiguraron según las nuevas necesidades contingentes: permanecer, trabajar y estudiar a diario, además de socializar y recrearse. Durante los confinamientos la vivienda se transforma en el componente tangible de la ciudad aumentada, donde las tecnologías ampliaron el ámbito doméstico permitiendo mantener cierta "normalidad" cotidiana. En los apartados anteriores abordamos la idea del silencio de las ciudades y de la transfiguración de lo urbano, procesos que se hicieron tangibles en pocos metros cuadrados: en la casa. Esto no solo evidencia las transformaciones (contingentes) de lo urbano, sino que da cuenta de la transdimensionalidad del habitar contemporáneo. La hiperrealidad digital amplía la experiencia de lo doméstico durante los intermitentes confinamientos - ya lo hacía con menor intensidad antes de la pandemia-, mientras el Orden Digital hace tangible la ciudad aumentada en nuestras propias casas desde el uso y apropiación social de la tecnología. Así, se habilita un nuevo espacio híbrido, transdimensional, que nos acerca al habitar convencional (aunque reconfigurado), que nos permite trabajar, estudiar, comercializar, proveernos de lo necesario para la vida cotidiana, entretenernos y socializar; o realizar manifestaciones políticas y vivenciar festividades. El Orden 
Digital hace de la casa y la vivienda la célula tangible de la ciudad aumentada. Bien cabe preguntarnos entonces: ¿cuáles de estos cambios transforman en el tiempo la vivienda y la concepción de lo doméstico? ¿Qué es lo que se mantiene y qué es lo que muta?

Todo esto que se percibe promisorio conlleva repensar las desigualdades prexistentes que la pandemia profundizó, como también las tensiones emergentes que emanan de estas transfiguraciones programáticas y conceptuales del habitar "privado". Las desigualdades de acceso a la vivienda como a lo digital expresan una doble expulsión social. La falta de una vivienda -0 el hacinamiento presente en los sectores vulnerados- sumada a la incapacidad técnica y cultural de poder acceder y pertenecer al Orden Digital expanden las brechas sociales, económicas, educativas y políticas.

Para el caso argentino, según el INDEC (2021), durante 2020 se registró un aumento del 3,1 \% en el acceso a internet en hogares, cifra total que asciende al $86 \%$. A pesar de los avances y políticas públicas implementadas, aún el $14 \%$ de hogares no tienen acceso a una conexión fija que posibilite mayores velocidades y menores costos. Esta diferencia se subsana -en algunos casos- por conexiones móviles. Aun así, según el mismo instituto, el 2 \% de la población no tiene acceso de ningún tipo. Esta situación se conjuga con 3.8 millones de familias con déficit habitacional (ACIJ, 2020) ${ }^{10}$, esto es, necesidades habitacionales insatisfechas; una cifra no consensuada, ya que incluyen aspectos cualitativos y cuantitativos disímiles. No obstante, dan cuenta de una asociación de factores que promueven la segregación y expulsión social en lo urbano, al tiempo que evidencian “nuevas” necesidades básicas, como lo es el acceso a internet.
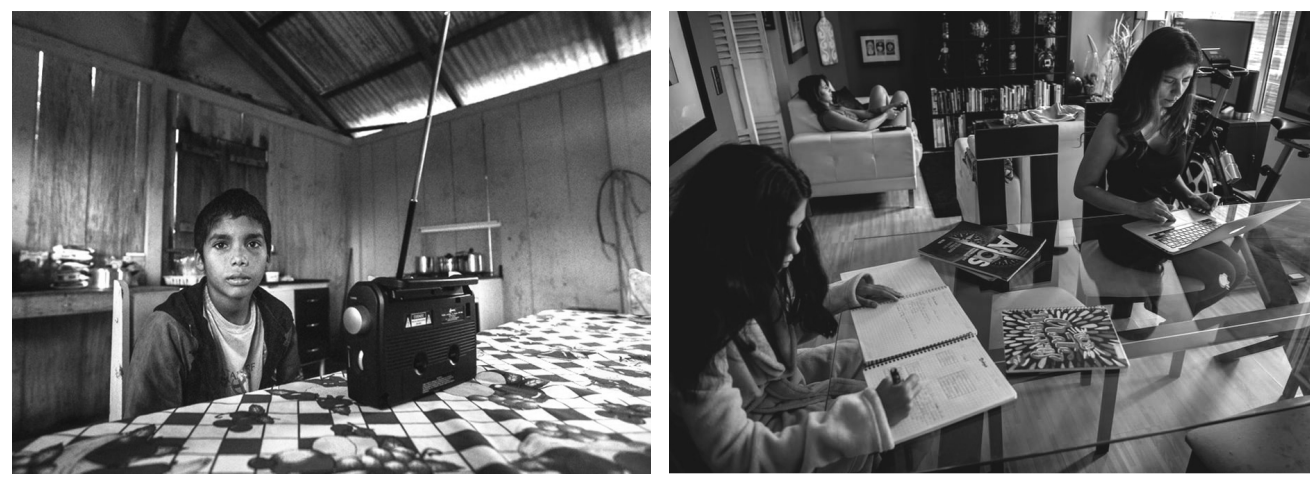

Figura 3. Desigualdades explícitas. Contrastes domésticos en el Orden Digital: dos maneras de habitar los flujos. Elaboración propia a partir de El Búho (2020) y Martín Bernetti-AFP (2020) 


\section{El espacio convergente}

Por último, como se argumentó al principio de este trabajo, la ciudad es en sí una hibridación constante entre componentes físicos-espaciales (urbs/ville) y socioculturales (civitas/cité), y ahora tecno-digitales, que se entrelazan y expanden desde otra topología urbana emergente: la metápolis, la ciudad aumentada. Este nuevo modo de habitar reconoce que las TIC y la automatización de los artefactos —en tanto discursos y herramientas-son intermediantes de lo urbano. La interacción con estas tecnologías hiperconectadas y su interdependencia con las personas configuran un nuevo sujeto social y urbano: el actante digital (Latour, 2008; Cortez Oviedo, 2020). Tanto sujetos humanos como no-humanos (TIC-A) se entrelazan en una interconstrucción dialéctica: un sujeto híbrido entre cuerpo y tecnología. Al interactuar con un dispositivo conectado a internet, responder y contestar mensajes, o acceder a la oferta algorítmica de nuestras redes sociales y de entretenimientos, se consuma esta relación semiótica e hibridación tecno-cultural. Ser actantes digitales (humanos y no-humanos) es estar entrelazado con el Orden Digital. No solo por la conexión física de sus tecnologías, sino también por sus discursos y consumos culturales; ser parte de sus tendencias, de sus lógicas y sentidos, es en definitiva ser parte de su tecno-cultura. Como todo proceso cultural, necesita un soporte y un territorio en el cual desarrollarse. Anteriormente, argumentamos que este es una hibridación constante entre componentes físicos, intangibles y tecnológicos: un espacio convergente.

Hablar de Espacio Convergente es referir a los archipiélagos que conforman la hiperrealidad digital, una constante interacción entre lo público y lo privado que expande no solo lo doméstico, sino también lo público —como espacio y construcción política-. Alcanza nuestras relaciones personales, laborales, sociales y políticas en la intangibilidad, pero también sus visibilidades físico-espaciales en lo urbano y lo doméstico. Es la red experiencial que conforma la ciudad aumentada, tantas como particularidades socioculturales y económicas expresen las personas. Representa la singularidad de la experiencia socio-urbana, que no solo se expresa en los espacios de la ciudad histórica, sino también dentro de nuestras viviendas.

Es el Espacio Convergente el que tensiona el habitar "privado". Es el que amplía los sentidos programáticos de la vivienda hipermoderna y promueve la domesticidad ubicua capaz de albergar y generar actividades que hasta hace poco eran ajenas a la casa. Es el que transfiere a lo doméstico la oficina, el aula de una escuela, la sala de cine y la mirada ajena que pone en jaque la privacidad. En lo urbano, este espacio se manifiesta reconfigurando el sentido de los espacios públicos como lugar y concepto, extendiéndolo a componentes 
intangibles y entrelazados cuyas dinámicas y prácticas sociales se expresan en la calle, las construcciones identitarias y en los conflictos de la ciudad histórica.

En este habitar de flujos, podemos inferir que el espacio convergente no es propio de esta contingencia sanitaria. Esta dialéctica espacial y líquida estaba ya presente en los procesos del espacio público contemporáneo, particularmente en aquellos con relación a la gamificación urbana $^{11}$. No obstante, es cierto que la COVID-19 trasladó y evidenció esta misma dinámica a los entornos domésticos, producto de la migración de actividades "urbanas" al ámbito privado: teletrabajo, teleducación, entretenimiento e interacción social. Estas convergencias de experiencias y ubicuidades hacen necesario no solo (re)pensar lo urbano y lo doméstico como conceptos y caracterizaciones, sino fundamentalmente desde un pensamiento proyectual emergente. ¿De qué manera el diseño resolverá esta dialéctica? ¿Qué recursos conceptuales necesitamos para pensar y construir nuevos tipos y tipologías de vivienda?

Estas miradas a escala posibilitaron un recorrido ondulante entre enfoques históricos y prospectivos que problematizaron vinculaciones y relaciones emergentes entre lo urbano, la COVID-19 y su proceso acelerado de digitalización. Se argumentó que esta pandemia promueve cambios en lo urbano, los espacios públicos y lo doméstico (aunque transfigurado), que requieren la reconfiguración de categorías instrumentales de la Arquitectura y el Urbanismo. Como analizamos, algunas de estas dinámicas eran prexistentes a la contingencia sanitaria, mientras que otras fueron su resultado directo. De igual modo, en el presente, se trata de interacciones que potencian o disminuyen las capacidades y el desarrollo humano en el hábitat de lo urbano, por lo cual requieren profundizar estudios al respecto. Tanto la ciudad histórica como ciudad aumentada, como la transformación de los espacios públicos y domésticos, articulados por la hiperrealidad digital del espacio convergente, afectan lo urbano como experiencia física, simbólica y estética. Finalmente, aquello que llamamos ciudad, ¿se ha transformado o desnaturalizado?

\section{Reflexiones finales}

Desde un sentido ontológico, se promovieron discusiones acerca de lo urbano, los espacios públicos y el habitar doméstico. Apoyados en la experiencia de la COVID-19, y desde los estudios previos sobre la cuarta revolución tecnológica, se plantearon interrogantes para el campo de la Arquitectura y el Urbanismo. A partir de esta mirada y contexto, lo urbano se expresa por una ciudad del aire, remota, holística, entrelazada por componentes
11. Con relación al uso y apropiación social de juegos geolocalizados que hicieron de las ciudades, y particularmente de sus espacios públicos físicosespaciales, sus entornos de acción y socialización; por ejemplo: Ingress (2012), Pokémon Go (2016) y Harry Potter Wizart Unite (2019). 
físicos-espaciales y socioculturales. Una ciudad (a)territorializada en la topología de la hiperrealidad del Orden Digital, alimentada por pulsos eléctricos, intercambios de bits, flujos sociales y consumos culturales mediados por lo digital.

Este nuevo habitar configura una ciudad aumentada, una metápolis, forma emergente de lo urbano, que requiere pensarse no solo en este contexto de pandemia y restricciones, sino como nuevo paradigma de aquello que llamamos ciudad, en tanto idea, espacio, lugar y relación social. Es una urbanidad capaz de generar otros entornos de intercambios socioculturales. La pandemia verifica que se puede prescindir de los cuerpos en las calles durante un tiempo, no así de las interacciones sociales, las que en este contexto encuentran otras formas y soportes donde desarrollarse y expresarse. No obstante, en caso de interiorizar esta contingencia sanitaria, la descorporización de lo urbano representa un desafío concreto para la ciudad histórica y sus vínculos sociales, estéticos y políticos.

Con el enmudecimiento de la ciudad histórica, lo digital se expresa como el lugar de los intercambios sociales, productivos y políticos, los que se transfiguran en una experiencia ubicua y alocalizada. Es una realidad expandida que se expresa por el espacio híbrido entre lo público, lo doméstico y lo urbano: un espacio convergente. Es a partir de su (con)vivencia como lugar y entorno ciberfísico que se unifican los archipiélagos de flujos de las personas: relaciones laborales, sociales, políticas y educativas, y también sus formas de producir, comerciar y ejercer ciudadanía. Es en esta interacción tecno-cultural entre personas, máquinas y discursos que se expresa un nuevo sujeto urbano: el actante digital, que a través de las pantallas intermedia en transformaciones de la interfaz urbana, social, económica y política de los urbanitas.

Se han planteado nuevas cuestiones en las ciudades del tercer milenio: la primera es la transformación de la relación sociedad-territorio (físico), que ha conformado lo urbano conocido en el siglo XX y que obliga a nuevas estrategias de supervivencia y adaptación individual y social. La segunda es la modificación del binomio integración-exclusión, ya que integrados y excluidos lo son ahora, además de por su pertenencia-localización en el sistema socioeconómico y en el territorio físico-espacial, por su pertenencia o localización entre los ricos y pobres en información, por su integración o no a la sociedad informacional.

De todas estas transformaciones, la espacial es la más evidente. Los espacios públicos, las calles y la vivienda se ven fuertemente afectados por estas dinámicas que relocalizan procesos entre lo material y lo intangible. Luego de esta contingencia, la ciudad, los espacios públicos y 
la vivienda representarán una entidad programática emergente. El campo urbano-arquitectónico se enfrentará así al desafío de poder traducirlo en proyecto arquitectónico y urbano.

Al fin de este ensayo, más que certezas, se buscó aportar inquietudes y prospecciones que sirvan como disparadores de una reflexión disciplinar. Como sugiere Souto de Mora (2015), se necesita pensar y construir una nueva disciplina; quizás una Arquitectura y un Urbanismo que además de la tectónica tangible, incorporen lo digital como información y materia emergente, más allá de lo instrumental, como nuevo territorio (GAUSA ET AL., 2002), para lo cual la transdisciplinariedad será clave.

¿De qué manera el proyecto incorporará este nuevo enfoque y materialidad? ¿Qué tipo de ciudad emerge de estas convergencias? ¿Qué reconfiguraciones socioculturales expresa? ¿Cuáles son sus premisas programáticas? Son algunos de los desafíos que representa el Orden Digital, y que esta pandemia hace evidentes en la oportunidad de afrontarlos. Se puede debatir el cómo, pero no puede seguir postergándose el para qué. Tanto el mundo globalizado ubicuo y el capitalismo de datos como los sesgos algorítmicos y las nuevas expulsiones sociales expresan un nuevo habitar. Desde esta reflexión, ¿cuánto de sus desafíos pueden incorporar la Arquitectura y el Urbanismo a su praxis proyectual?

\section{Referencias bibliográficas}

Ascher, F. (2004). Los nuevos principios del urbanismo. Alianza.

Aumont, J. (2013). La Imagen. Paidós.

AA. VV. (2020). Cities of Silence. Extraordinary views of a shutdown world. Ed. TeNeues.

Baudrillard, J. (2002). Cultura y simulacro. Kairós.

Benedikt, M. (2020). Architecture beyond experience. Applied Research \& Design.

Benjamin, W. (2003). La obra de arte en la época de la reproductividad técnica. Editorial Ítaca.

Borja, J. \& Castells, M. (2006). Local y global. La gestión de las ciudades en la era de la información. Taurus.

Carrión, F. (2020, 10-13 de noviembre). La transformación de la comunicación local en tiempo de pandemia [ponencia]. Conferencia IFAP- UNESCO. Brechas, deudas y logros: lo que la pandemia revela sobre la Sociedad del Conocimiento en América Latina. Modo virtual. https://www.youtube.com/watch?v=RNqs8VTNzw\&t=3537s 
Castells, M. (2006). La sociedad red. Una visión global. Alianza.

Coll López, J. (2018). Determinación vs indeterminación en el espacio doméstico. Los límites de la flexibilidad. Rita 10, 64-72.

Cortez Oviedo, P. S. (2020). Espacios públicos en el Orden Digital. El rol de las TIC en sus usos y apropiaciones sociales [tesis doctoral]. Editorial UNC. https://rdu. unc.edu.ar/handle/11086/15596

Echeverría, J. (1994). Telépolis. Destino.

Fernández, R. (2014, 17-21 de marzo). Teoría, crítica y gestión de la arquitectura, la ciudad y el territorio [Seminario]. Conferencia Pensamiento proyectual, Córdoba, Argentina.

Finquelievich, S. (Comp.); Feldman, P.; Girolimo, U. \& Odena, M. B. (2019). El futuro ya no es lo que era. Editorial Teseo Press. https://www.teseopress.com/ elfuturoyanoesloqueera/

Finquelievich, S. (2016): I-Polis. Ciudades en la era de Internet. Diseño.

Gausa, M.; Guallart, V.; Müller W.; Morales, J.; Porras, F. \& Soriano F. (2002). Diccionario Metápolis de arquitectura avanzada, ciudad y tecnología en la sociedad de la información. Actar.

Geertz, C. (2003). Las interpretaciones de la cultura. Gedisa.

Lévy, P. (2004). Inteligencia colectiva. Por una antropología del ciberespacio. OPS.

López García, G. (Ed.). (2005). El ecosistema digital. Modelos de comunicación, nuevos medios y públicos en internet. Publicaciones de la Universidad de Valencia.

Katz, R. (2015). El ecosistema y la economía digital en américa latina. Editorial Ariel y Fundación Telefónica.

Habermas, J. (2009). Historia y crítica de la opinión pública. Gustavo Gili.

Hénaff, M. (2016). La ciudad que viene. Lom ediciones.

Jay, M. (2003). Campos de fuerza. Entre la historia intelectual y la crítica cultural. Regímenes escópicos de la modernidad. Paidós.

Lotman, Y. (2019). La semiósfera. Semiótica de la cultura y del texto. Fondo Editorial.

Masotta, C. (abril de 2018). Antropología de lo visual. En Seminario Maestría en antropología social FFyH -UNC. Curso llevado a cabo en Córdoba, Argentina.

Morris, A. (2018). Historia de la forma urbana. Gustavo Gili.

Tsatsou, P. \& Elaluf-Calderwood, S. (2007). Theoretical and Methodological Foundations for a Knowledge Base of Regulatory Issues. Trust in Digital Business Ecosystems. https://www.researchgate.net/publication/235758962 
Rainie L. \& Wellman B. (2012). Networked: The New Social Operating System. The MIT Press.

Rybczynski, W. (2013) La casa. Historia de una idea. Nerea.

Sarquis, J. (2011). Arquitectura y modos de habitar. Ediciones de la U.

Scolari, C. (2013). Narrativas Transmedia: Cuando Todos los Medios Cuentan. Deusto.

Sennett, R. (2019). Construir y habitar. Ética para la ciudad. Anagrama.

Silva, A. (2006). Imaginarios Urbanos. Arango editores.

Silva, A. (Comunicación personal, s/f 2016). Ciudad de aire. https://www.eltiempo.com/opinion/columnistas/armando-silva/ciudad-de-aire-armando-silva-columnista-el-tiempo-53532

Simak, C. (1988). Ciudad. Minotauro.

Solà-Morales, M. (2006). Las formas del crecimiento urbano. Ediciones UPC.

Souto de Moura, E. (Comunicación personal, 26 de julio de 2015). Los arquitectos van a tener que construir una nueva disciplina. Plataforma arquitectura. https://www. plataformaarquitectura.cl/cl/770817/souto-de-moura-los-arquitectos-vana-tener-que-construir-una-nueva-disciplina

Wainwright, O. (Comunicación personal, 11 de febrero de 2020). The countryside is where the radical changes are: Rem Koolhaas goes rural. The Guardian. https:// www.theguardian.com/artanddesign/2020/feb/11/rem-koolhaas-ruralcountryside-the-future-guggenheim

\section{Informes documentales}

CABASE (2020). Cabase internet index 2020. Estado de Internet en Argentina y la RegiónPrimer semestre. Oficina Cámara Argentina de Internet. https://www. cabase.org.ar/wp-content/uploads/2020/07/CABASE-Internet-Index-1erSemestre-2020.pdf

INADI (2021). Informe anual 2020. Oficina Instituto Nacional contra la Discriminación, la Xenofobia y el Racismo de Argentina. https://www.argentina.gob. ar/sites/default/files/17032021_coordinacion_de_investigaciones_y_ observatorios.pdf

INDEC (2021). Accesos a internet. Cuarto trimestre de 2020 [Informe técnico Vol. 5, núm. 4.]. Oficina Instituto Nacional de Estadísticas y Censos de Argentina. https:// www.indec.gob.ar/ 